\title{
Tackling Fluid Overload in a High-transporter Diabetic Patient on Continuous Ambulatory Peritoneal Dialysis
}

\author{
Elwaleed A M Elhassan*, Osman-mahir M A Mahir, Hisham M Ali, Aymun I Mobarak, Maha A \\ Hummeida, Abeer abdallah, Afraa Abdelwahid
}

Ribat Center for Regular Peritoneal Dialysis, Ribat University Hospital, Khartoum, Sudan

\begin{abstract}
Introduction: Diabetic patients on continuous ambulatory peritoneal dialysis (CAPD) are more prone to fluid overload than non-diabetic patients, but the use of hypertonic glucose solutions to improve their ultrafiltration (UF) may hamper their glycemic control. Maintaining euvolemia in such patients may be tricky and needs special care.

Case report: A 72 year old diabetic and hypertensive patient presented with severe fluid overload shortly after initiation of CAPD despite producing more than one liter of urine per day. He only achieved modest ultrafiltration (UF) during the day, and had a negative UF during the long hypertonic night dwell. Peritoneal equilibration tests (PET) confirmed that he was a high transporter, and his weekly Kt/V was found to be 1.36. Since automated peritoneal dialysis (APD) is not yet available in Sudan, the PD prescription was modified to comprise five short cycles during the day, including two short hypertonic daytime dwells, and a dry abdomen at night. This approach succeeded in improving his fluid status, but required the addition of intra-peritoneal soluble insulin to his regular subcutaneous insulin in order to achieve acceptable blood sugar control. After 8 months his residual renal function (RRF) had declined remarkably and he began to suffer from intermittent fluid overload of variable degrees. Nevertheless, we managed to maintain him satisfactorily on CAPD for 14 months.
\end{abstract}

Conclusion: Simple measures such as omitting the night dwell and using five short cycles during the day, including two short hypertonic dwells, can be effective in controlling fluid overload in diabetic patients who have a high transporter status.

\footnotetext{
* Corresponding author; Division of Renal Diseases and Hypertension, University of Colorado Health Sciences Center, P.O.Box 6508, Aurora, CO 80045, USA.

E mail: Elwaleed.Elhassan@UCHSC.edu
}

Key words: CAPD, diabetes mellitus, fluid overload, residual renal Function

\section{Introduction}

Fluid overload is a leading cause of mortality and morbidity in CAPD patients as it incurs a cumulative cardiovascular burden. A high proportion of chronic kidney disease (CKD) patients presents with established cardiovascular disease at the time of dialysis initiation; notably left ventricular hypertrophy, coronary artery disease and congestive cardiac failure [1].

Volume homeostasis in CAPD patients is affected by several factors including patient compliance, salt and fluid intake, residual renal function (RRF), the use of loop diuretics and angiotensin converting enzyme inhibitors (ACEi), tonicity of dialysis solutions, and membrane transport characteristics [1-5].

Special attention should be paid to diabetic patients since they are more prone to fluid overload than non-diabetic patients despite the use of more hypertonic glucose solutions and, as a consequence, higher peritoneal ultrafiltration (UF) and higher total fluid removal [5]. The use of hypertonic glucose solutions to improve UF may also hamper glycemic control.

Here we present a simple but effective approach that we adopted for the management of an overloaded diabetic patient with high transporter status.

\section{Case report}

A 72 year old male with long standing uncontrolled diabetes mellitus, hypertension and ischemic heart disease presented to our unit with stage 5 CKD. A Tenckhoff catheter was inserted, and CAPD was initiated two weeks later with the conventional four 2-liter exchanges, utilizing the maximum tonicity $(4.25 \%)$ in the long night dwell. Shortly thereafter he presented with severe fluid overload that was evident by increasing weight, raised 
blood pressure, pulmonary congestion, lower limb edema and hyponatremia.

Initial assessment revealed that he was eating a liberal diet, including free salt intake and plenty of fluids. He was not taking a diuretic despite having good RRF, with urine output exceeding 1 liter per day. He had a modest daytime UF, and negative UF following the long night dwell, i.e. the morning drain was less than 2 liters. Random blood sugar (RBS) readings ranged between 250 and $400 \mathrm{mg} / \mathrm{dl}$, being maintained on total subcutaneous mixtard insulin of $50 \mathrm{IU} /$ day. His glycated hemoglobin (HbA1C) level was 9.5\%.

We implemented a diet restricted in salt with a maximal fluid intake of one liter per day. We added $40 \mathrm{mg}$ of Lisinopril daily with the purpose of preserving the RRF as well as $120 \mathrm{mg}$ of oral furosemide twice daily to augment diuresis. He showed a good initial response to these measures; within two weeks signs of hypervolemia had notably lessened and serum sodium concentration rose from 115 to $129 \mathrm{mmol} / \mathrm{l}$. However, complete euvolemia was hard to attain and he continued to have some degree of fluid overload.

Adequacy and peritoneal equilibration tests (PET) were performed; he turned out to be a high transporter with a weekly $\mathrm{Kt} / \mathrm{V}$ of 1.36 and a creatinine clearance of 47 liter/week $/ 1.73 \mathrm{~m}^{2}$. These results had demonstrated that he had been under-dialyzed according to the minimum $\mathrm{Kt} / \mathrm{V}$ recommended by the International Society for Peritoneal Dialysis (ISPD) of 1.7, while the high transporter state largely explained the fluid overload and the negative UF of the long dwell. Dialysis intensification by increasing the volume of exchanges, the number of exchanges per day, or both, as well as the use of icodextrin (a poorly absorbed glucose polymer) should have been helpful in achieving better Kt/V and UF for this patient. Unfortunately, neither cycler based automated PD nor icodextrin are currently available in Sudan.

Compliance with the PD prescription was verified and emphasized. It was subsequently modified to include five cycles a day, including two short hypertonic daytime dwells. This approach achieved some improvement in the patient's fluid status, but the long night dwell kept on draining negatively. It was hence decided to omit the night dwell; the prescription was again modified to comprise five short cycles during the day, including two short hypertonic daytime dwells, with dry abdomen at night.

This approach succeeded to some extent in improving his fluid status However, using two bags of concentrated glucose solutions per day rendered glycemic control more difficult. Thus, intra-peritoneal soluble insulin was introduced at a dose of $40 \mathrm{IU}$ per cycle in addition to the subcutaneous mixtard insulin. Consequently, readings decreased and $\mathrm{HbA1C}$ dropped over a three-month period from $9.5 \%$ to $8.3 \%$.

The patient continued to present intermittently with fluid overload as his urine output declined steadily to about $200 \mathrm{ml} /$ day over a period of 8 months. Transfer to hemodialysis was discussed with the patient and family, but they were reluctant and preferred to continue on CAPD.

Until the time of writing this report, he has been on CAPD for 14 months with intermittent variable degrees of fluid overload.

\section{Discussion}

All PD patients should ideally be edema free; moreover, they should be normotensive with the minimal use of antihypertensive medications except for reno-cardioprotective agents.

All possible factors should be addressed in a systematic manner in order to accurately identify and treat the cause of fluid overload in any particular patient. Special attention should be paid to diabetic patients since they are more prone to fluid overload than non-diabetics despite the use of more hypertonic glucose solutions and, as a consequence, higher peritoneal UF and higher total fluid removal [5]. The use of hypertonic glucose solutions to improve UF may hamper glycemic control, cause hyperlipidemia, weight gain and accumulation of glucose degradation products (GDPs) and advanced glycation end-products (AGEs) which are potentially damaging to the peritoneal membrane [6].

If hypervolemia is present, catheter malfunction should first be ruled out, e.g. leaks, hernias, block or malposition. Other simple measures, such as ensuring patient compliance with PD prescription need to be addressed. Those steps are quite often effective in solving the issue.

Although fluid overload contributes to hypertension in CAPD patients, little attention has been paid to the role of excess salt and fluid intake in compromising blood pressure control. Cheng et al [3] suggested that RRF may not be as important as expected in maintaining good volume status; in their study, patients with higher sodium and fluid removal had higher blood pressure despite having higher RRF. They suggested that restricting salt and fluid intake might be more important for better blood pressure control in CAPD patients than religiously guarding the RRF.

Restricting salt and water intake can effectively treat fluid overload in diabetic and non-diabetic peritoneal 
dialysis patients. Dietary salt and fluid restriction may also help reduce the use of hypertonic glucose solution and thus facilitate blood glucose control in diabetic patients undergoing peritoneal dialysis [5].

The use of high dose loop diuretics facilitates water and salt removal in the presence of RRF [4], but special attention should be paid to patients with declining RRF in whom urine output may not be sufficient to achieve adequate fluid and salt removal in response to loop diuretics.

UF profile should be studied starting with the long dwell, as this may be helpful in tailoring the PD prescription according to the patients needs. If the UF is poor or negative an extra cycle may be added at night, the concentration of dialysis solution may be increased or icodextrin may be used. If the short dwells also drain poorly, use of concentrated solutions, increase in the number of cycles per day or increase in the fill volume in heavy individuals should be considered. These modifications should be applied bearing in mind the assessment for PET and PD adequacy [1].

\section{Conclusion}

Achieving adequate control of fluid overload may be difficult in diabetic patients who have high transporter status, particularly in developing countries where APD is not widely available. Simple measures such as omitting the night dwell and using five short cycles during the day, including two hypertonic dwells, may be effective in improving the fluid status of those patients.

\section{References}

1. Abu-Alfa AK, Burkart J, Piraino B, Pulliam J, Mujais S. Approach to fluid management in peritoneal dialysis: A practical algorithm. Kidney Int Suppl. 2002 Oct;(81):S8S16.

2. Chen W, Cheng LT, Wang T. Salt and fluid intake in the development of hypertension in peritoneal dialysis patients. Ren Fail. 2007;29(4):427-32.

3. Cheng LT, Chen W, Tan W, Wang T. Residual Renal Function and volume control in peritoneal dialysis patients. Nephron Clin Pract. 2006;104(1):c47-54.

4. Krediet RT. Dry body weight: water and sodium removal targets in PD. Contrib Nephrol. 2006;150:10410.

5. Gan HB, Chen MH, Linholm B, Wang T. Volume control in diabetic and non diabetic peritoneal dialysis patient. Int Urol Nephrol. 2005; 37(3):595-9.

6. Sitter T, Sauter M. Impact of glucose in peritoneal dialysis: saint or sinner? Perit Dial Int. 2005 Sep-Oct; 25(5):415-25. 